\title{
SEÑAL ACÚSTICA Y REDESCRIPCIÓN DE GONGROCNEMIS (GONGROCNEMIS) MUNDA BRUNNER VON WATTENWYL, 1895 (ORTHOPTERA: TETTIGONIIDAE)
}

\section{ACOUSTIC SIGNAL AND REDESCRIPTION OF GONGROCNEMIS (GONGROCNEMIS) MUNDA BRUNNER VON WATTENWYL, 1895 (ORTHOPTERA: TETTIGONIIDAE)}

\author{
GEOVANY J. FERNÁNDEZ-AZUARA ${ }^{1}$, LUDIVINA BARRIENTOS-LOZANO ${ }^{1 *}$, AURORA Y. ROCHA- \\ SÁNCHEZ ${ }^{1 *}$, AleJANdro ZALDÍVAR-RIVERÓN ${ }^{2}$, PEDro ALMAGUER-SIERRA ${ }^{1}$, AlFONSO CORREA- \\ SANDOVAL ${ }^{1}$

\footnotetext{
${ }^{1}$ Tecnológico Nacional de México-Instituto Tecnológico de Ciudad Victoria-División de estudios de Posgrado e Investigación. Boulevard Emilio Portes Gil No.1301. Ciudad Victoria, C.P. 87010, Tamaulipas, México. <biol.geofa@hotmail.com>; <ludivinab@yahoo.com>; <auro_3110@hotmail.com>; <almagavetec@hotmail.com>; <alf_correas@hotmail.com> Universitaria, Copilco, Coyoacán. A. P. 70-233, C.P 04510. Cd. de México. <azaldivar@ib.unam.mx>

*Autor corresponsal: <ludivinab@yahoo.com>; <auro_3110@hotmail.com>
} \\ ${ }^{2}$ Universidad Nacional Autónoma de México-Instituto de Biología. Colección Nacional de Insectos. 3er. Circuito exterior s/n. Cd.
}

Recibido: 04/08/2019; aceptado: 09/01/2020; publicado en línea: 10/02/2020 Editor responsable: Magdalena Cruz

Fernández-Azuara, G. J., Barrientos-Lozano, L., Rocha-Sánchez, A. Y., Zaldívar-Riverón, A., Almaguer-Sierra, P., Correa-Sandoval, A. (2020) Señal acústica y redescripción de Gongrocnemis (Gongrocnemis) munda Brunner von Wattenwyl, 1895 (Orthoptera: Tettigoniidae). Acta Zoológica Mexicana (nueva serie), 36, 1-14. https://doi.org/10.21829/azm.2020.3612230

RESUMEN. En los ortópteros de la superfamilia Tettigonioidea las señales acústicas son de especial interés, ya que juegan un papel importante en el apareamiento y están involucradas en el reconocimiento intraespecífico macho-hembra. La señal acústica de llamado permite al macho atraer individuos del sexo opuesto a corta o larga distancia. En este trabajo se presenta la señal acústica de llamado de Gongrocnemis (Gongrocnemis) munda (Tettigoniidae: Pseudophyllinae) y se hace una breve redescripción de esta especie, en particular de la hembra, no descrita previamente. Ejemplares de $G$. $(G$.) munda se recolectaron en San

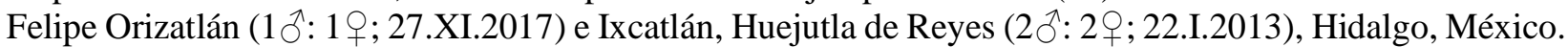
G. (G.) mexicana se recolectó en Cd. del Maíz, San Luis Potosí (1 + ; 19.VII.2003). Los machos producen la señal acústica de llamado principalmente durante la noche y está compuesta por pares de equemas que se emiten ininterrumpidamente por periodos de una hora o más. Machos de $G$. (G.) munda producen $6.0 \pm 0.9$ (d.e) equemas/min ( $n=30 \mathrm{~min}$; tres machos, $10 \mathrm{~min}$ cada uno). La duración por equema-par es de $3.97 \pm$ (d.e) $1.2 \mathrm{~s}(2.7-8.0 \mathrm{~s})$ ( $n=30$ equemas-pares). El análisis de frecuencia muestra la mayor liberación de energía de $16-40 \mathrm{kHz}$, con una fi pico de $20-38 \mathrm{kHz}$. La hembra es similar al macho, pero más robusta. El color general del cuerpo es verde oliváceo con matices color crema y manchas marrón obscuro. La longitud es de $32.7 \pm 1.5 \mathrm{~mm}(n=3)$. Esta especie habita selva mediana y bosque de encino, se encuentra generalmente sobre la copa o el tronco de los árboles. Durante el día permanecen quietos y ocultos, siendo activos por la noche.

Palabras clave: Pseudophyllinae; bioacústica; taxonomía 
Fernández-Azuara, G. J., Barrientos-Lozano, L., Rocha-Sánchez, A. Y., Zaldívar-Riverón, A., Almaguer-Sierra, P., Correa-Sandoval, A. (2020) Acoustic signal and redescription of Gongrocnemis (Gongrocnemis) munda Brunner von Wattenwyl, 1895 (Orthoptera: Tettigoniidae). Acta Zoológica Mexicana (nueva serie), 36, 1-14. https://doi.org/10.21829/azm.2020.3612230

\begin{abstract}
In the Orthoptera of the superfamily Tettigonioidea acoustic signals are of special interest since they play an important role in the process of pair formation and are directly involved in intraspecific male-female recognition. The calling signal usually allows the male to attract individuals of the opposite sex at short or long distance. In this work, we present the acoustic signal of Gongrocnemis (Gongrocnemis) munda (Tettigoniidae: Pseudophyllinae) and provide a brief redescription of this species, in particular of the female not formally described previously. Specimens of $G$. (G.) munda were collected in San Felipe

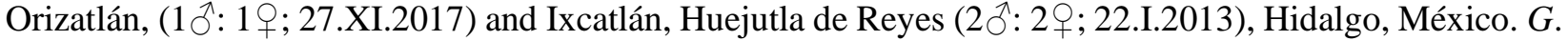
(G.) mexicana was collected in Ciudad del Maíz, San Luis Potosí (1 ; 19.VII.2003). Males produce the calling acoustic signal mainly at night, and it is composed of paired-echemes. The acoustic signal may be produced uninterruptedly for long periods, $1 \mathrm{~h}$ or more. Males of $G$. (G.) munda produce $6.0 \pm 0.9$ (s.d) echemes per minute ( $n=30 \mathrm{~min}$; three males, $10 \mathrm{~min}$ each). Duration per paired-echeme is $3.97 \pm$ (s.d) $1.2 \mathrm{~s}$ (2.7-8.0 s) ( $n=30$ echemes). Frequency analysis showed the highest energy release between $16-40 \mathrm{kHz}$, with a fi peak in the range of 20-38 kHz. The female is similar to the male, but more robust. The general body color is olive-green, with cream spots and brown marks. The average length is $32.7 \pm 1.5 \mathrm{~mm}(n=3)$. The ecosystem this species lives on is tropical evergreen and oak forest; it is found usually on the foliage or trunk of trees. During the day, adults remain quiet and hidden, being active at night.
\end{abstract}

Key words: Pseudophyllinae; bioacoustics; taxonomy

\title{
INTRODUCCIÓN
}

En los ortópteros de la superfamilia Tettigonioidea las señales acústicas son de especial interés, ya que juegan un papel importante en el apareamiento y están directamente involucradas en el reconocimiento intraespecífico macho-hembra (Siegert et al., 2013; Dutta et al., 2017). El canto o señal de llamado permite al macho atraer individuos del sexo opuesto a corta o larga distancia y se produce de forma espontánea sin que el macho esté en contacto con individuos conespecíficos (machos o hembras) (Heller et al., 2004, 2017). Por otra parte, los machos pueden emitir una señal acústica de cortejo que se produce generalmente cuando las hembras están próximas y entran en contacto físico, por ejemplo, mediante las antenas, incluso durante la cópula (Alexander, 1967; Robinson \& Hall, 2002; Dutta et al., 2017). Las señales acústicas, por ser especie-específicas y tener una función importante en el sistema de apareamiento, son relevantes no solo en estudios evolutivos, sino también como una herramienta de gran utilidad en la delimitación de especies, particularmente en grupos de organismos que usan este sistema de comunicación, como es el caso de los Tettigonioidea. El género Gongrocnemis (Orthoptera: Tettigoniidae) fue descrito por Redtenbacher, 1895 (citado en Brunner von Wattenwyl, 1895). Este es un grupo de Pseudophyllinae tropicales, cuya distribución se extiende desde el norte de México a América del Sur (norte de Argentina). Actualmente, el género está compuesto por tres subgéneros [Gongrocnemis (Gongrocnemis) Redtenbacher, 1895; G. (Melanocnemis) Beier, 1954 y G. (Schizocnemis) Beier, 1954] y 28 especies (Cigliano et al., 2019). Brunner von Wattenwyl (1895) ofrece los caracteres distintivos del género Gongrocnemis según se expone a continuación. Cabeza corta, globosa, no hundida en vista frontal. El fastigio no supera el margen de las escrobas antenales, antenas marrón oscuro, anuladas. Pronoto tuberculado color marrón, con márgenes postero-laterales color negro con forma de trenza. Élitros color marrón oscuro, alas color marrón jaspeado. Fémures marrón jaspeado. Placa supranal pequeña, triangular, ranurada. Cercos cortos, adelgazándose en forma gradual distalmente, punta curva. Lamina subgenital cónica, ligeramente emarginada, estilos cortos y cilíndricos. Gongrocnemis (Gongrocnemis) munda Brunner von Wattenwyl, 1895 es endémica de México, ocurre en la Provincia Biogeográfica del Golfo de México, recolectada en Córdoba y Orizaba, Veracruz (Cigliano et al., 2019). 
Hay poca información disponible sobre G. (G.) munda, aparte de la breve descripción original realizada por Brunner von Wattenwyl, 1895 en la cual solo se describe el macho recolectado en Córdoba, Veracruz. Algunos autores hacen referencia a esta especie y mencionan la existencia de hembras en las colecciones, no obstante, la hembra no ha sido descrita formalmente (Saussure et al., 1893-1899; Kirby, 1906; Beier, 1954, 1962; Hollier \& Maehr, 2012). En el presente trabajo se aporta información sobre la señal acústica producida por los machos de $G$. (G.) munda, se describe por primera vez la hembra y se redescribe e ilustra el macho.

\section{MATERIALES Y MÉTODOS}

Ejemplares adultos de G. (G.) munda, se recolectaron en el Estado de Hidalgo, México, municipios de San Felipe Orizatlán: 1ठ: 19, 27.XI.2017, 21.169319 N, -98.625783 O, 140 msnm; e Ixcatlán, Huejutla de Reyes: $2{ }^{\lambda}:$ : 2 , 22.I.2013, 21.0667 N, -98.5500 O, $844 \mathrm{msnm}$. Ambas localidades forman parte de la huasteca hidalguense. Los adultos se transportaron vivos al laboratorio de Ecología del Tecnológico Nacional de México-Instituto Tecnológico de Cd. Victoria (TecNM-ITCV) y se mantuvieron separados, machos y hembras, en jaulas entomológicas de $30 \times 30 \times 30 \mathrm{~cm}$, para que estuvieran sexualmente receptivos y facilitar la producción y grabación de la señal acústica. Como alimento se les proporcionó lechuga y hojas de durazno cada 2 días. La grabación de la señal acústica se realizó por la noche. La sala de prueba de sonido mide $3 \times 3 \mathrm{~m}$ y está aislada con paneles de espuma insonorizados de $5 \mathrm{~cm}$ de espesor. Se grabaron tres machos por un periodo de 1 hora cada uno, aproximadamente. Para grabar la señal se utilizó un micrófono Dodotronic-Ultramic (UM250K, Italia), longitud $130 \times 20 \mathrm{~mm}$ de diámetro, $50 \mathrm{~g}$ de peso, con una frecuencia de muestreo de $250 \mathrm{kHz}$, un rango de frecuencia de $125 \mathrm{kHz}$, resolución 16 bits, temperatura $27 \pm 2{ }^{\circ} \mathrm{C}$, colocado a una distancia de $50 \mathrm{~cm}$; el micrófono ultrasónico con entrada-USB se conectó a una lap-top utilizada exclusivamente para grabar las señales acústicas. La señal acústica se analizó con el programa Cool Edit Pro 2.1 (Syntrillium Software Corporation, Phoenix, Arizona, Estados Unidos de América), tamaño FFT 1024. Posterior al análisis la señal se filtró para elaborar el oscilograma. Para describir la señal acústica se siguió la terminología propuesta por Ragge y Reynolds (1998), Stumpner et al. (2013), Heller et al. (2004, 2015). Canto de llamado: canto producido por un macho solo, generalmente aislado de otros, cuando está sexualmente maduro. Equema: ensamble o grupo de primer orden de sílabas que representa una unidad de sonido-canto. Sílaba: sonido producido durante un ciclo individual y completo de apertura y cierre de las tegmina. Hemi-sílaba: sonido producido por un movimiento unidireccional de las tegmina en este caso durante la apertura. Pulsación ("Pulse"): sonido aislado, producido generalmente por la estimulación de uno o varios dientes del peine estridulador. Intervalo Mayor-IMa: intervalo de silencio o casi, que sigue a un tren de pulsaciones de una, dos o más sílabas; en este trabajo denota el intervalo entre equemas-pares. Intervalo Menor-IMe: en este trabajo, señala el intervalo de silencio o casi, que hay entre equemas 1 y 2 en secuencias de equemas-pares. Se midió la tasa de repetición de secuencias de equemas por min (t. r. equemas/min) en el canto de llamado ( $n=30 \mathrm{~min}$; tres machos, 10 min cada uno); la duración de equemas-pares ( $n=30$ equemas-pares); la duración de equemas 1 y 2 en secuencias de equemas-pares ( $n=50$ equemas-pares); el intervalo mayor (IMa) entre secuencias de equemas-pares ( $n=30$ equemas pares); el intervalo menor (IMe) entre equemas 1 y 2 en secuencias de equemas-pares ( $n=50$ equemas); el espectro de frecuencia y la frecuencia $(f i)$ pico.

Para confirmar los caracteres morfológicos de $G$. (G.) munda se revisaron ejemplares de $G$. $(G$.) bivittata en la Orthoptera Species File online (http://orthoptera.speciesfile.org/Common/basic/Taxa.aspx? TaxonNameID=1140239) y 1 q de G. (G.) mexicana recolectada en Cd. del Maíz, San Luis Potosí, 19.VII. $2003,22^{\circ} 01.914^{\prime} \mathrm{N}$ y $100^{\circ} 14.868^{\prime} \mathrm{O}, 1100 \mathrm{msnm}$.

Las fotografías de los caracteres morfológicos de diagnóstico se tomaron con un microscopio estereoscópico marca MOTIC-SWZ-168739® equipado con cámara digital de 10 mp, con aumento de 10X. 
Depósito de material examinado. Colección L. Barrientos-Lozano, Tecnológico Nacional de MéxicoInstituto Tecnológico de Cd. Victoria (TecNM-ITCV), Tamaulipas, México.

\section{RESULTADOS}

Señal acústica. Machos y hembras de G. (G.) munda viven en selva mediana y bosque de encino sobre follaje de la copa o el tronco de los árboles. Los ejemplares se recolectaron sobre Quercus crassifolia Humb. \& Bonpl. Durante el día permanecen quietos y mimetizados con el follaje o el tronco de los árboles; son de hábitos nocturnos. El canto de llamado se escucha como un chirrido suave de baja intensidad. La señal acústica de llamado de $G$. (G.) munda está compuesta predominantemente por secuencias de equemas-pares (Fig. 1), ocasionalmente se producen equemas individuales. En una muestra de 16 min del canto de llamado, se emitieron 88 equemas (78 equemas-pares y 10 individuales). La tasa de repetición (t.r. $/ \mathrm{min}$ ) es de $6.0 \pm$ (d.e) 0.9 equemas/min ( $n=30 \mathrm{~min}$; tres machos, $10 \mathrm{~min}$ cada uno). Cada par de equemas tiene una duración de $3.97 \pm 1.2 \mathrm{~s}(2.7-8.0 \mathrm{~s} ; n=30$ equemas-pares). El IMa entre equemas-pares tiene una duración de $7.07 \pm$ $1.00 \mathrm{~s}$ (5.28-9.47 s; $n=30$ equemas pares). La duración del primer equema en cada dúo es de $469 \pm 35 \mathrm{~ms}$ (378-528 ms; $n=50$ equemas), el IMe entre el equema 1 y el equema 2 en equemas-pares es de $2.60 \pm 0.778$ $\mathrm{s}(1.71-5.19 \mathrm{~s} ; n=50$ equemas) y la duración del segundo equema es de $481 \pm 35 \mathrm{~ms}(420-560 \mathrm{~ms} ; n=50)$. El número de sílabas en los equemas 1 y 2 en el dueto de equemas es muy consistente, en el equema 1 el número promedio de sílabas es de $21 \pm 3$ (16-26 sílabas; $n=30)$; mientras que en el equema 2 el número promedio de sílabas es de $22 \pm 3$ (17-27 sílabas; $n=30)$. El análisis de frecuencia de $G$. (G.) munda (Fig. 2a, b) muestra un espectro $>90 \mathrm{kHz}$, la mayor cantidad de energía se libera entre 16 a $40 \mathrm{kHz}$ y la fi pico entre 20-38 kHz. Solo se grabó el canto de llamado, ya que no se produjo otro tipo de señal acústica durante el periodo de grabación. La señal acústica es en general muy consistente. Al iniciar el canto el macho puede producir señales de un solo equema. No obstante, conforme el macho va practicando uniformiza la señal y el patrón de ésta es de equemas-pares, principalmente.

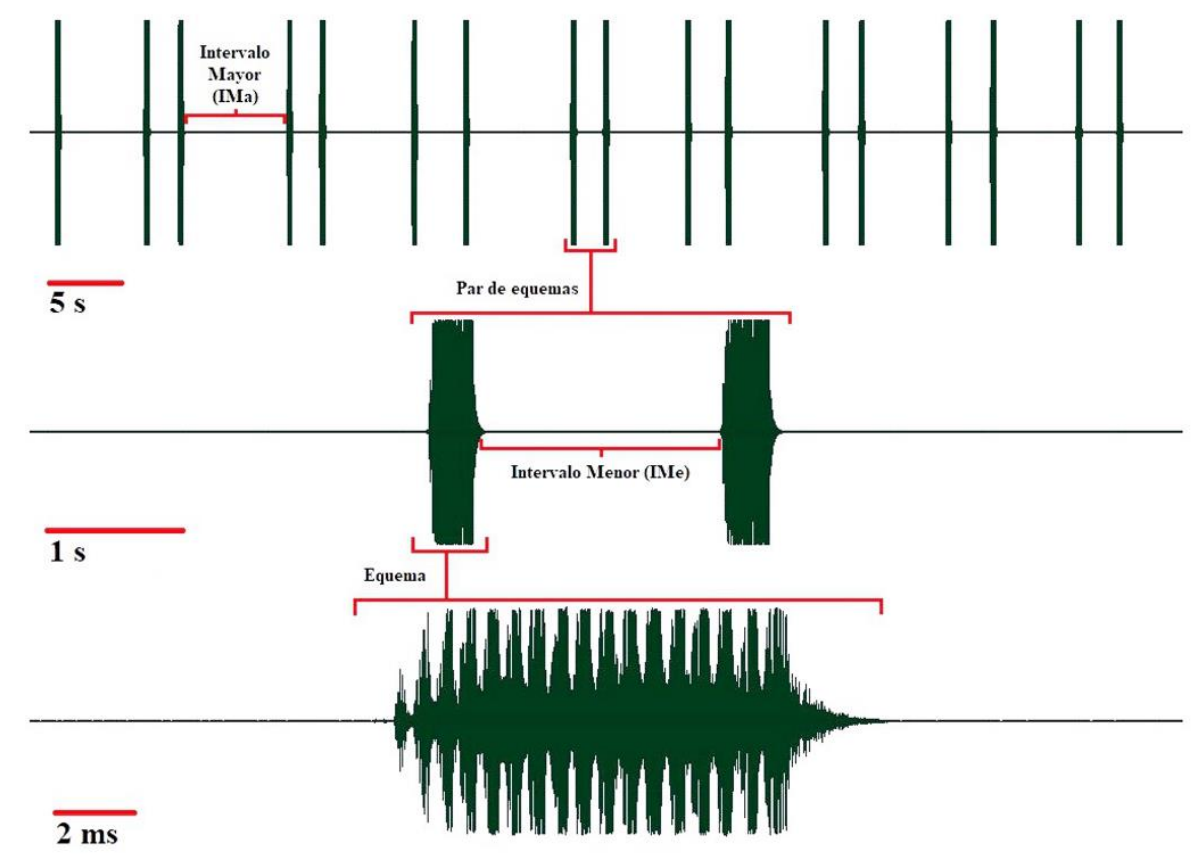

Figura 1. Oscilograma de la señal acústica de llamado de un macho de Gongrocnemis (G.) munda de San Felipe Orizatlán, Hidalgo, México. 

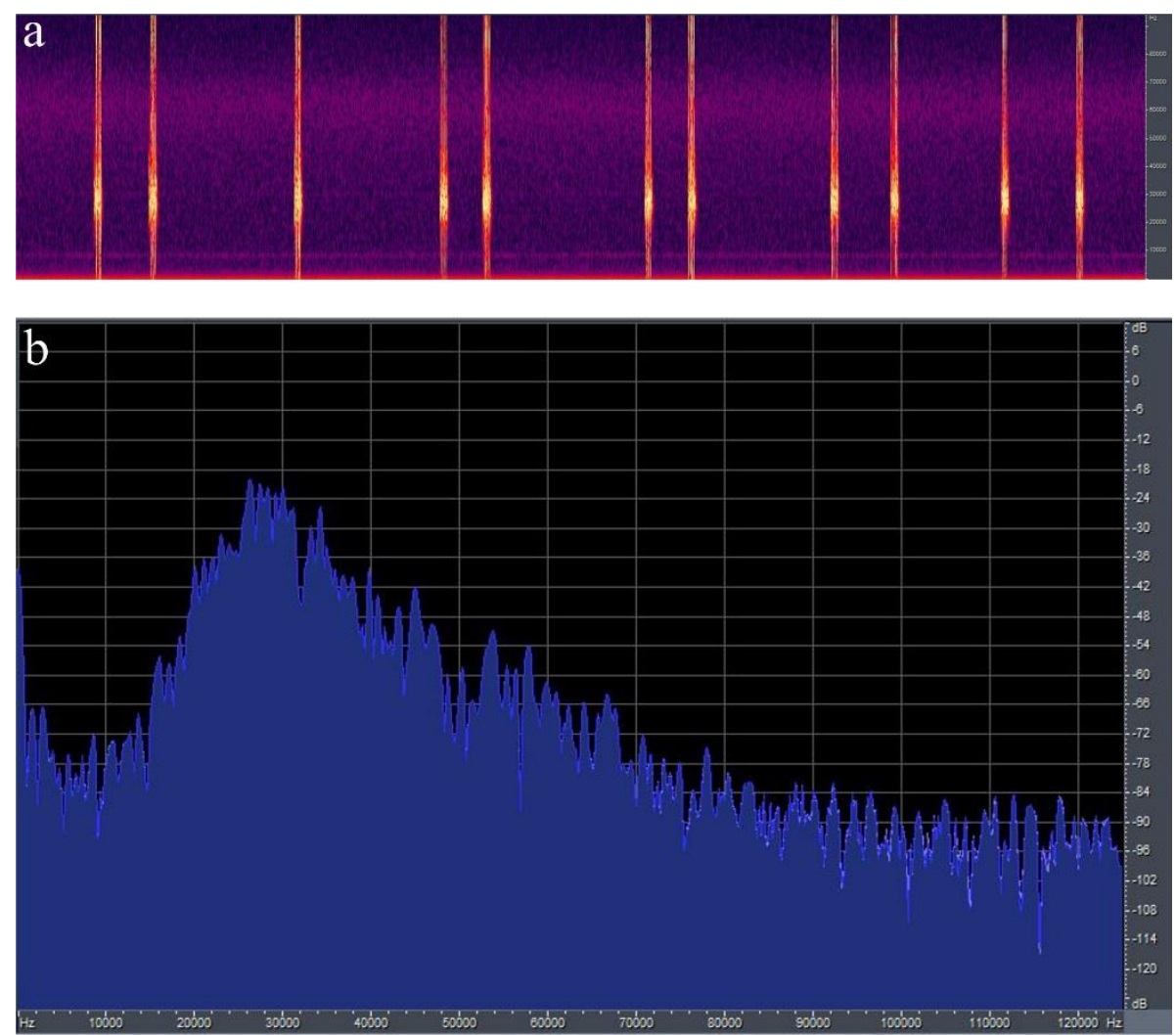

Figura 2. a) Sonograma y espectro de potencia en la señal acústica de llamado de un macho de Gongrocnemis ( $G$.) munda de San Felipe Orizatlán, Hidalgo, México. b) Espectro de frecuencia y fi pico en la señal acústica de llamado de un macho de Gongrocnemis (G.) munda de San Felipe Orizatlán, Hidalgo, México.

Redescripción de Gongrocnemis (Gongrocnemis) munda Brunner von Wattenwyl, 1895.

Figs. 3-23.

Material examinado: $1 \delta$ : 1 ․ Estado de Hidalgo, San Felipe Orizatlán, 27.XI.2017, 21.169319 N, 98.625783 O, 140 msnm. G. de J. Fernández-Azuara. 20 : 2ㅇ. Ixcatlán, Huejutla de Reyes, 22.I.2013, 21.0667 N, -98.55 O, 844 msnm. L.A. Hernández-Cortés. Col. L. Barrientos-Lozano, TecNM-ITCV.

Diagnosis. $G$. (G.) munda puede confundirse con $G$. (G.) bivittata Brunner von Wattenwyl, 1895 y/o $G$. (G.) mexicana (Saussure, 1859), ya que las tres especies ocurren en la Provincia Biogeográfica del Golfo de México. Sin embargo, machos (Figs. 3-15) y hembras (Figs. 16-23) de G. (G.) munda pueden diferenciarse de G. (G.) bivittata (Orthoptera Species File: http://orthoptera.speciesfile.org/Common/ basic/Taxa.aspx?TaxonNameID=1140224), por el pronoto densamente granuloso, gránulos más grandes y conspicuos en la prozona, con un tubérculo prominente sobre la porción media del margen anterior y uno menos prominente en el margen posterior; el ángulo que une el margen posterior del pronoto-metazona con los lóbulos laterales es mucho más pronunciado posteriormente que en $G$. (G.) bivittata. Adicionalmente, en las hembras el plato subgenital (Fig. 20) es distalmente más ancho y el ángulo proximal -en la incisión distal del plato subgenital- es también más amplio, el ovipositor (Fig. 21) es más recto que en $G$. $(G$.) bivittata. Con referencia a $G$. (G.) mexicana (Figs. 24-30), machos y hembras de G. (G.) munda pueden diferenciarse fácilmente por el plato subgenital; en los machos es mucho más ancho y la emarginación distal es apenas un escote (Fig. 12), mientras que en $G$. (G.) mexicana (http://orthoptera.speciesfile.org/Common/basic/ShowAllImages.aspx) es en forma de U-moderadamente 
profunda. En hembras, el pronoto, el plato subgenital y el ovipositor pueden separarlas fácilmente (Figs. 17, 20, 21 vs Figs. 24, 29, 30).
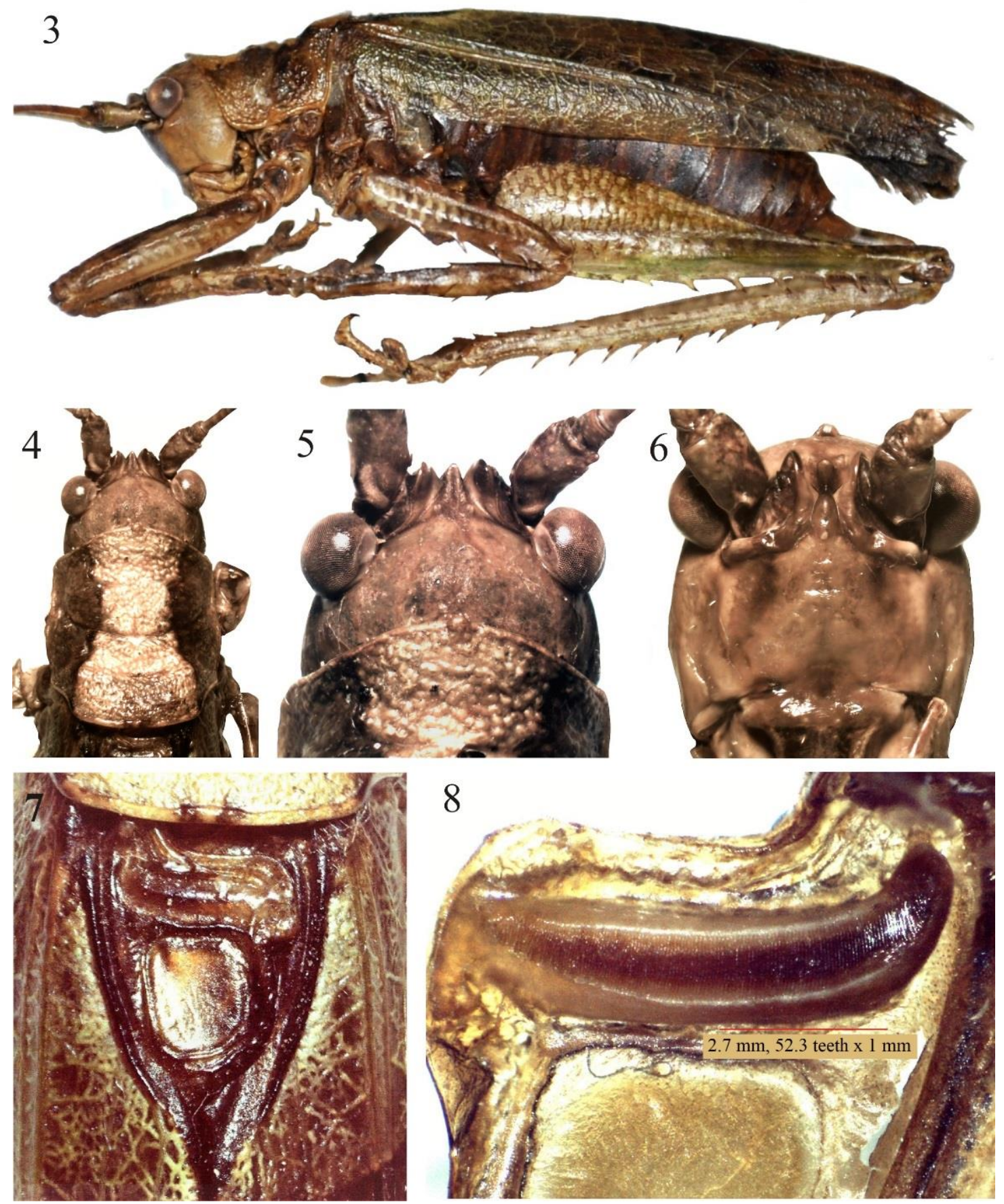

Figura 3-8. Gongrocnemis (G.) munda, Macho: 3) habitus. 4) cabeza y pronoto, vista dorsal. 5) fastigio del vertex vista dorsal. 6) fastigio frontal. 7) aparato estridulador. 8) peine estridulador. 

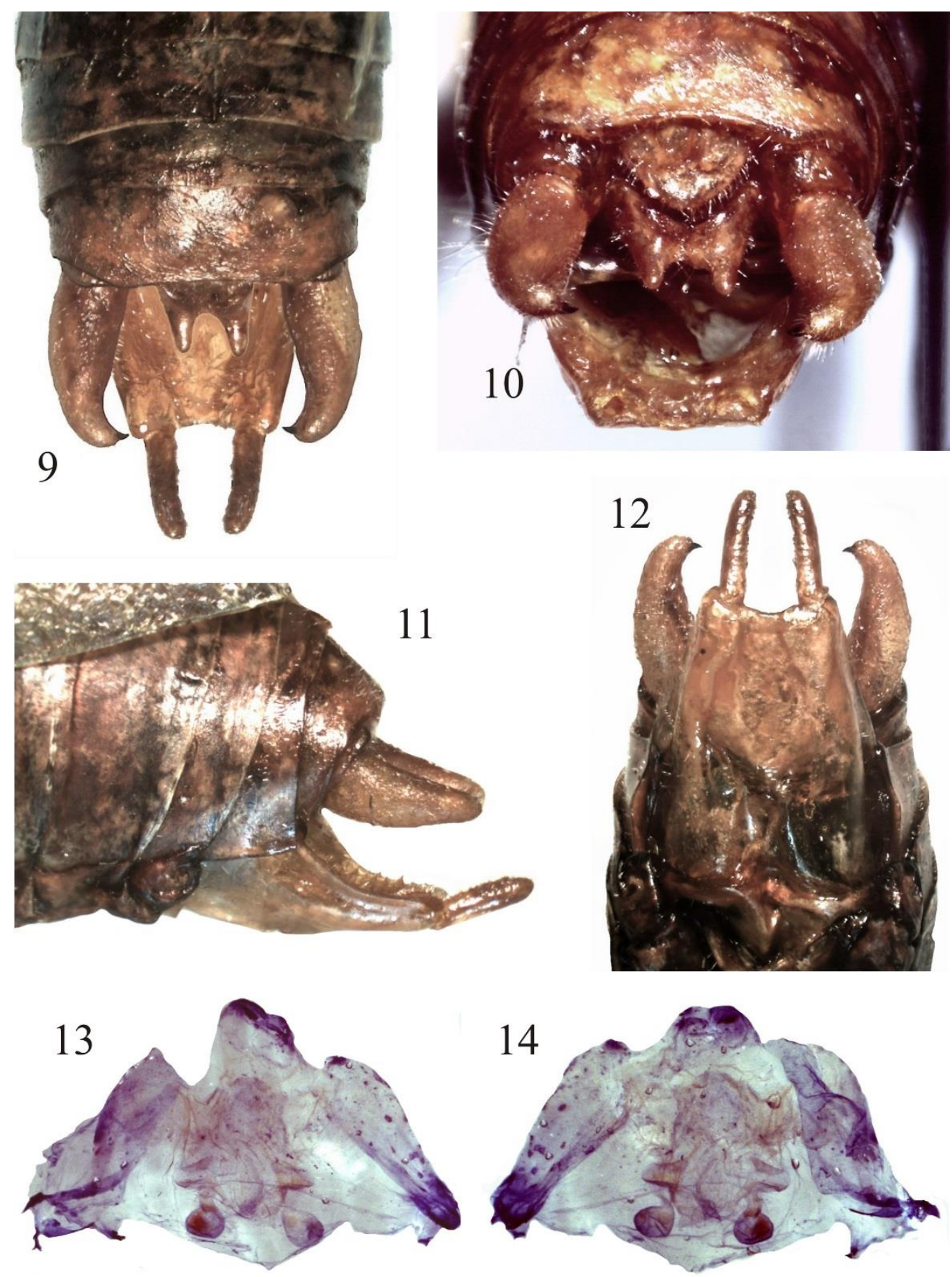

Figura 9-14. Gongrocnemis (G.) munda, Macho. 9) cercos y epiprocto vista dorsal. 10) plato supranal y epiprocto, vista frontal. 11) cercos y plato subgenital, vista lateral. 12) plato subgenital, vista ventral. 13) genitalia interna, vista dorsal. 14) genitalia interna, vista ventral.

Redescripción del macho de $G$. (G.) munda (Figs. 3-15). Color general del cuerpo (Figs. 3, 15) verdeoliváceo con manchas blancas en margen superior de las tegmina; pronoto dorsalmente color blanco-crema, con dos franjas marrón oscuro dorso-laterales, una a cada lado (Figs. 4, 15); aparato estridulador (Figs. 7, 15) color marrón, márgenes prácticamente color negro; margen superior de las tegmina -en reposo- con línea punteada color marrón obscuro (Figs. 7, 15). Antenas color crema, con segmentos marrón oscuro intercalados (Fig. 15). Fastigio del vertex (Fig. 5) surcado longitudinalmente, en la porción proximal con 
un tubérculo a cada lado visible y de moderado tamaño, distalmente no rebasa los sockets antenales. Fastigio frontal (Fig. 6) robusto y casi en contacto con el fastigio del vertex. Pronoto (Figs. 4, 15) dorsalmente color blanco-crema, márgenes laterales color marrón oscuro, tuberculado -tubérculos más conspicuos en la prozona-, márgenes anterior y posterior con un tubérculo prominente en la porción media, lóbulos laterales color verde oliva, cuando están vivos, marrón claro con matices marrón oscuro en ejemplares de colección (Figs. 3, 15). Aparato estridulador (Figs. 7, 15) color marrón claro con márgenes marrón obscuro a negro. Peine estridulador (Fig. 8) con longitud de $2.7 \mathrm{~mm}$ y 141 dientes, aproximadamente. Tegmina (Figs. 3, 15) color verde-olivo, con manchas color blanco-crema y oscuro en el margen superior. Patas jaspeadas con color verde-oliva y marrón obscuro. Fémur anterior, medio y posterior sin espinas en el margen ventral interno; fémur anterior, medio y posterior con cuatro (tres grandes y una pequeña, con punta color negro) y ocho espinas (seis grandes con punta color negro, dos pequeñas color amarillo), respectivamente, en el margen ventral externo. Tibias anterior, media y posterior con el siguiente número de espinas en los márgenes ventral interno y externo, respectivamente: 5, 6; 7, 7; 7, 9. Placa supranal (Fig. 10) pequeña y subtriangular. Epiprocto (Figs. 9, 10) sub-rectangular, distalmente con una incisión profunda en forma de U. Cercos (Figs. 9-12) robustos proximalmente, después del primer tercio se adelgazan gradualmente, en el último tercio se curvan hacia dentro para terminar en una uña gruesa y conspicua, color marrón-oscuro a negro. Placa subgenital (Fig. 12) sub-rectangular, la porción distal levemente emarginada, dando la apariencia de un escote ligero. Estilos (Figs. 9-12) de tamaño mediano -comparados con otras especies, p. ej., G. (G.) mexicana- y de forma cilíndrica. Genitalia interna como se muestra en las figuras 13 y 14.

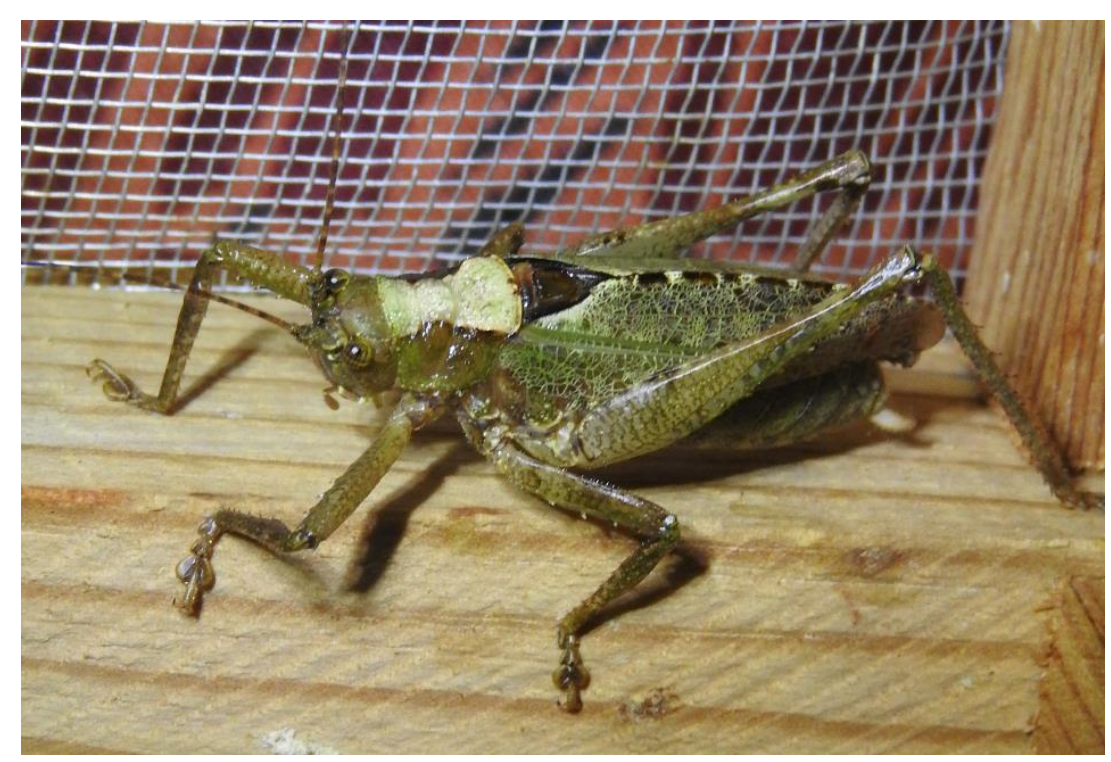

Figura 15. Gongrocnemis (G.) munda, Macho, vivo.

Descripción de la hembra de G. (G.) munda (Figs. 16-23). En apariencia general similar al macho (Figs. 16, 22, 23). Fastigio del vertex (Figs. 17, 18) sub-triangular color marrón-crema; fastigio frontal (Fig. 19) color marrón -distalmente más claro- más robusto que en el macho. La porción central de la cara (Fig. 19), entre el fastigio frontal y el clípeo, es de color marrón, genas color blanco-crema. Antenas (Figs. 16, 22, 23) color blanco-crema con segmentos y ánulos color marrón-oscuro intercalados. Pronoto (Figs. 17, 22, 23) color blanco-crema dorsalmente, rugoso y tuberculado, tubérculos más conspicuos en la prozona, con dos bandas longitudinales color marrón, una a cada lado, el surco transversal principal corta profundamente los lóbulos laterales, margen anterior proyectándose ligeramente hacia delante con un tubérculo prominente en la porción media, margen posterior emarginado y ligeramente redondeado, con dos manchas de color marrón y un tubérculo en la poción media, este último menos prominente que el del margen anterior. Fémur anterior 
con cuatro espinas en el margen ventral externo y la tibia con seis espinas en el margen ventral interno y siete en el externo. El fémur medio presenta cuatro espinas en el margen ventral externo, la tibia seis en el margen ventral interno y seis en el externo. Fémur posterior armado con ocho espinas en el margen ventral externo, tibia con 12 espinas en el margen ventral interno y siete a ocho en el externo. Plato subgenital (Fig. 20) de forma sub-triangular, emarginación distal en forma de V-amplia. Cercos (Fig. 21) grandes, bastante anchos, curvándose hacia dentro, pilosos, con punta delgada; en vista lateral. Ovipositor (Fig. 21) largo (23 $\mathrm{mm}$ de longitud), semi-recto y bastante ancho, la porción media proximal es color blanco-crema jaspeada con marrón obscuro, la mitad distal es color marrón obscuro, margen superior débilmente serrulado, sin denticulaciones en el margen ventral, ápice agudo; en vista lateral.

Medidas (mm). Machos. Longitud del cuerpo, desde el margen anterior -porción media del vertex- al final del abdomen, 31. Longitud del pronoto, 8. Longitud de las tegmina, 32. Longitud del fémur anterior, 10. Longitud del fémur medio, 11. Longitud del fémur posterior, 23. Hembras. Longitud del cuerpo, desde margen anterior -porción media del vertex- al final del abdomen, $32.7 \pm 1.5 \mathrm{~mm}(n=3)$. Longitud del pronoto, 8.8. Longitud de las tegmina, 35. Longitud del fémur anterior, 12. Longitud del fémur medio, 12. Longitud del fémur posterior, 24. Longitud del ovipositor, 23.

Distribución. Ejemplares de G. (G.) munda se recolectaron en la Región Huasteca del Estado de Hidalgo, México, en San Felipe Orizatlán y Huejutla de Reyes. $G$. (G.) mexicana se recolectó en Cd. del Maíz, San Luis Potosí.

Hábitat. Gongrocnemis $(G$.$) munda es arbórea. Habita en selva mediana y bosque de encino.$

\section{DISCUSIÓN}

En esta contribución se caracteriza el comportamiento acústico, en particular el canto de llamado, de Gongrocnemis $(G$.) munda y se redescribe esta especie. No hay antecedentes sobre la señal acústica de $G$. (G.) munda, ni sobre ninguna otro taxa miembro del género Gongrocnemis Redtenbacher (Cigliano et al., 2019), por lo que ésta sería la primera aportación al estudio de las señales acústicas de esta especie y del grupo. La señal acústica es un canto estereotipado, compuesto predominantemente de equemas-pares, la $f i$ pico de 20-38 kHz, cae en el rango de otros Pseudophyllinae que producen señales acústicas en el rango audible (< de $20 \mathrm{kHz}$ ) y ultrasónico (> de $20 \mathrm{kHz}$ ) (Montealegre-Zapata et al., 2003; Baker et al., 2019). Se describe por primera vez la hembra de $G$. (G.) munda y se redescribe e ilustra el macho. En la descripción original de esta especie (Brunner von Wattenwyl, 1895), solo se describió el macho. Varios autores mencionan a G. (G.) munda, sin embargo, no se hace la descripción de la hembra (Saussure et al., 18931899; Kirby, 1906; Beier, 1954, 1962; Hollier \& Maehr, 2012). La distribución de G. (G.) munda está reportada para el Golfo de México, en Córdoba y Orizaba, Veracruz (Brunner von Wattenwyl, 1895; Saussure et al., 1893-1899; Cigliano et al., 2019), por lo que este sería el primer reporte para la Huasteca de Hidalgo, México. G. (G.) mexicana se distribuye también en el Golfo de México, en Córdoba y Orizaba, Veracruz (Saussure, 1859; Cigliano et al., 2019); Barrientos-Lozano et al. (2013) reportan esta especie para el Estado de San Luis Potosí sin mencionar la localidad, la cual aquí se indica es Ciudad del Maíz, San Luis Potosí. Los miembros del género Gongrocnemis, son insectos grandes poco conocidos. Esto último debido tal vez a su habilidad para mimetizarse con el follaje o el tronco de las plantas hospederas. De acuerdo con Cigliano et al. (2019) se reconocen 28 especies para este género, de éstas, 11 especies se reportan para México. La información disponible para la mayoría de los taxa que componen el género es muy pobre, las descripciones originales son breves y para muchas de las especies se ha descrito solo el macho o la hembra (Brunner von Wattenwyl, 1895; Saussure et al., 1893-1899; Piza, 1980). Gongrocnemis es un género muy diverso, endémico para el continente americano y requiere una revisión profunda. 

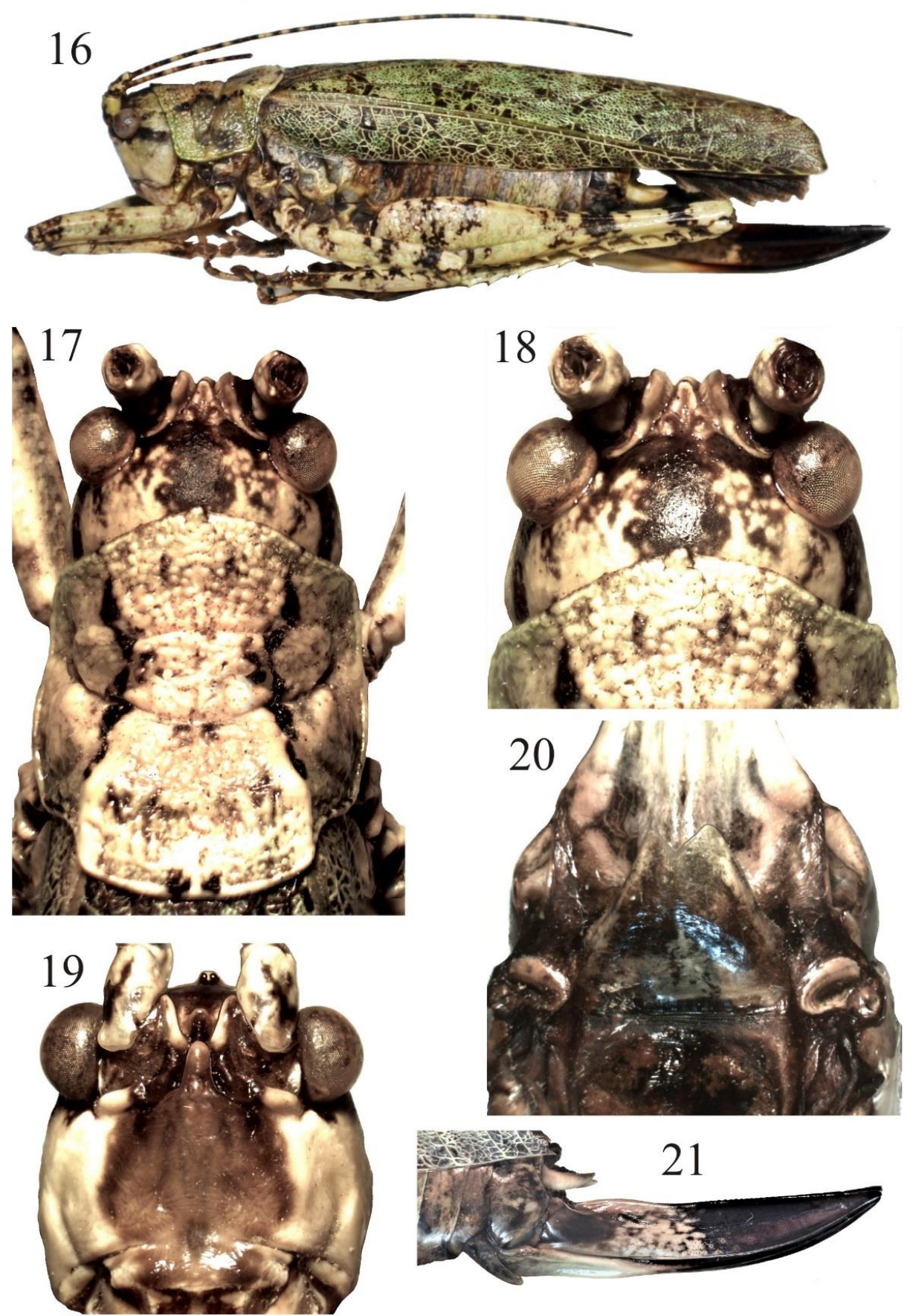

Figura 16-21. Gongrocnemis (G.) munda, Hembra. 16) habitus. 17) cabeza y pronoto, vista dorsal. 18) fastigio del vertex. 19) fastigio frontal. 20) plato subgenital, vista ventral. 21) ovipositor, vista lateral.

\section{CONCLUSIONES}

Se aporta información inédita sobre comportamiento acústico y se redescribe a Gongrocnemis $(G$.) munda. Se proporcionan caracteres de diagnóstico para $G$. (G.) munda y $G$. (G.) mexicana. Este representa tal vez el primer aporte al estudio del comportamiento acústico de uno de los miembros del género Gongrocnemis. En virtud de la diversidad del género y lo poco que se conoce sobre éste, se recomienda continuar con su estudio y llevar a cabo una revisión del mismo. 
AgradeCimiEnTOS. Al Consejo Nacional de Ciencia y Tecnología (CONACYT) por el apoyo proporcionado a GJFA a través del Programa Nacional de Becas (Beca de Doctorado No. 415264). Al Tecnológico Nacional de México-Instituto Tecnológico de Cd. Victoria, por el apoyo proporcionado para realizar trabajo de campo. Al CONACYT por el apoyo económico a través del proyecto CONACYT/CB/2013/0219979.

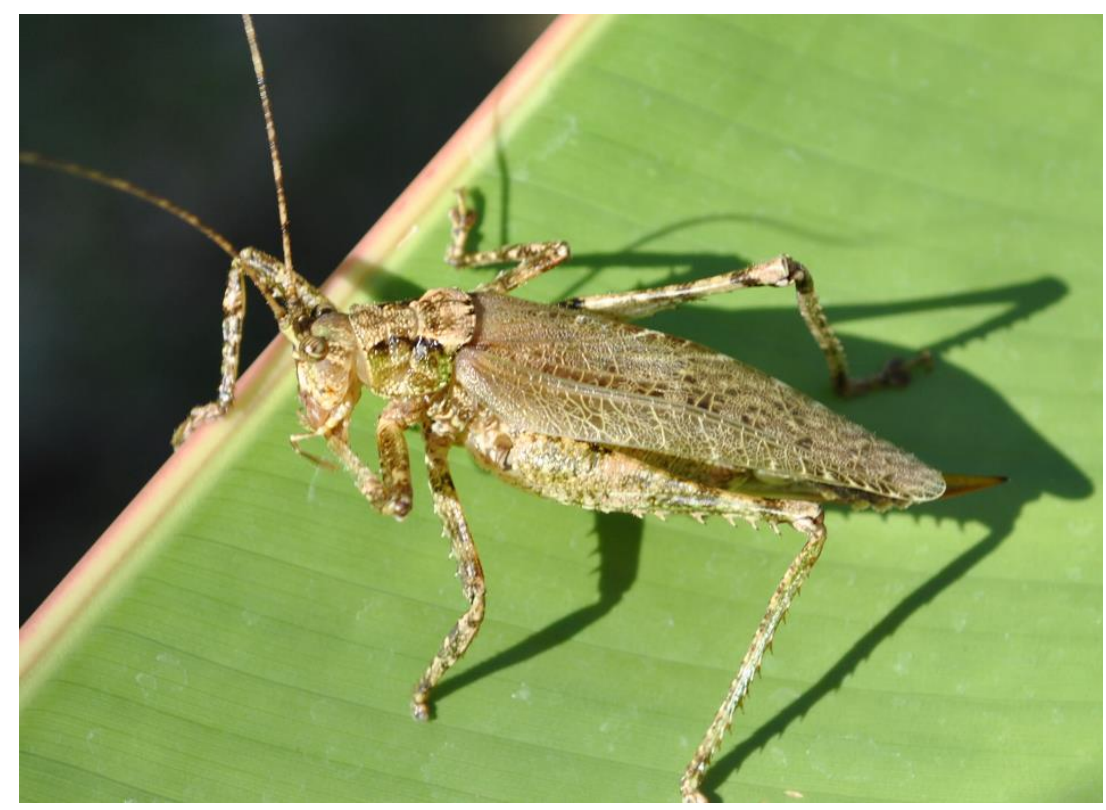

Figura 22. Gongrocnemis (G.) munda. Hembra, viva.

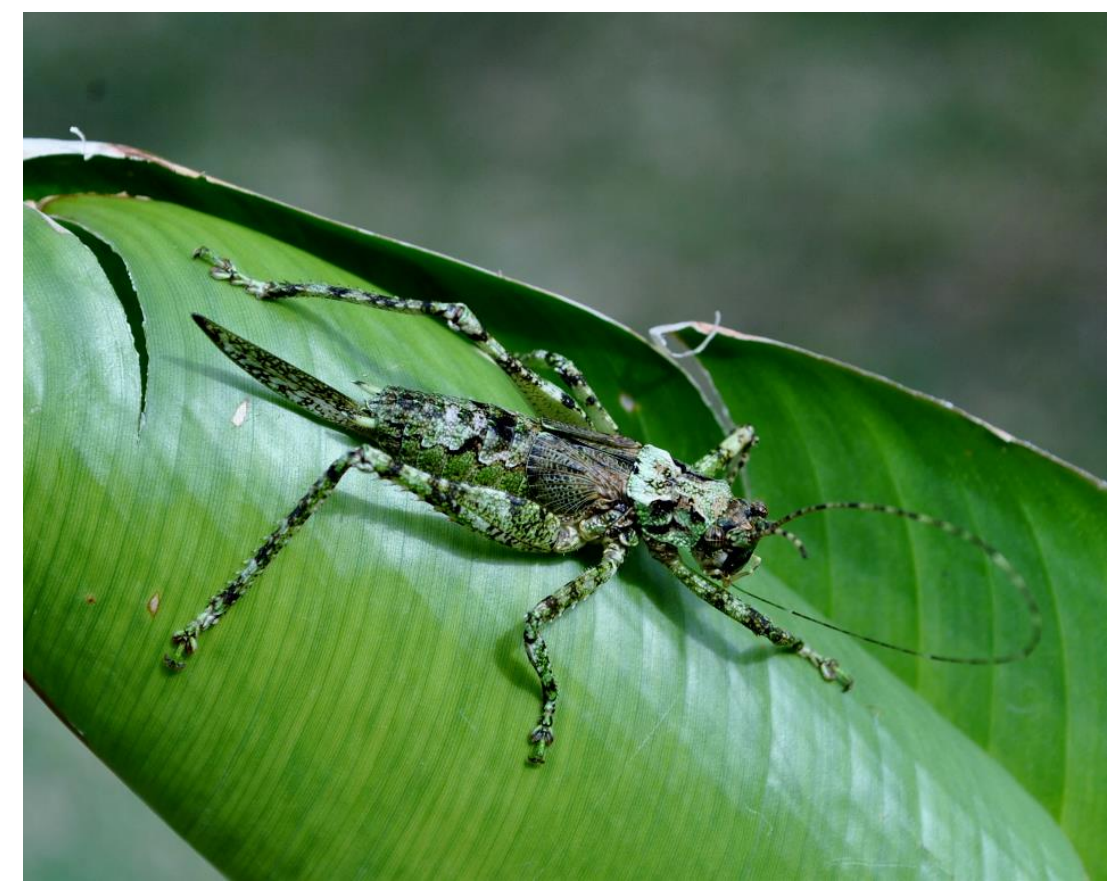

Figura 23. Gongrocnemis (G.) munda. Hembra, ninfa, viva. 

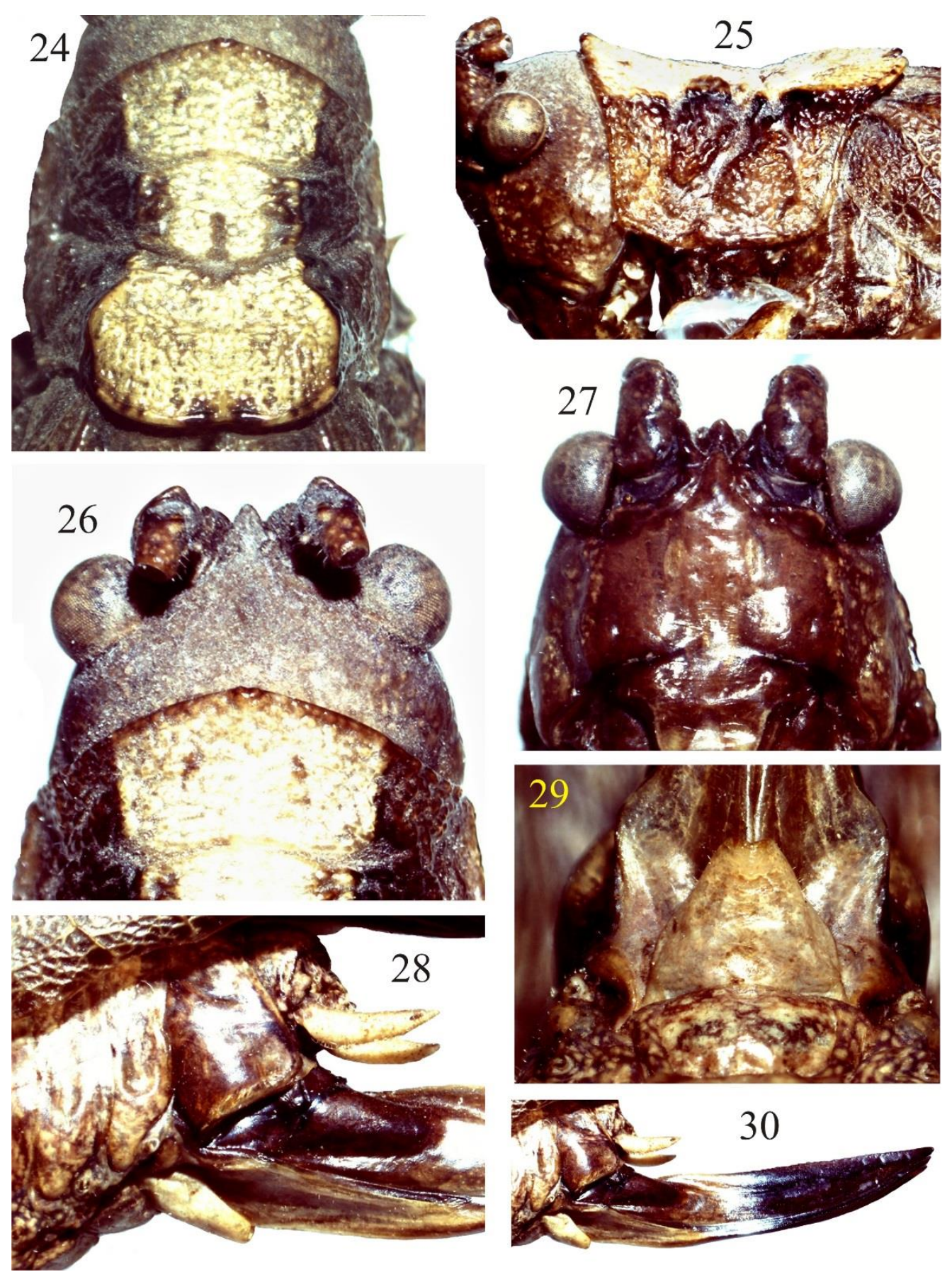

Figura 24-30. Gongrocnemis (G.) mexicana. Hembra. 24) pronoto, vista dorsal. 25) pronoto, vista lateral. 26) fastigio del vertex, vista dorsal. 27) fastigio frontal. 28) cercos y plato subgenital, vista lateral. 29) plato subgenital, vista ventral. 30) ovipositor. 


\section{LITERATURA CITADA}

Alexander, R. D. (1967) Acoustical communication in arthropods. Annual Review of Entomology, 12, 495526.

Baker, A. A., Jonsson, T., Aldridge, S., Montealegre-Zapata, F. (2019) Complex wing motion during stridulation in the katydid Nastonotus foreli (Orthoptera: Tettigoniidae: Pseudophyllinae). Journal of Insect Physiology, 114, 100-108.

https://doi.org/10.1016/j.jinsphys.2019.03.005

Barrientos-Lozano, L., Rocha-Sánchez, A. Y., Buzzetti, F. M., Méndez-Gómez, B. R., Horta-Vega, J. V. (2013) Saltamontes y Esperanzas del Noreste de México. Guía Ilustrada. Dirección General de Educación Superior Tecnológica. Instituto Tecnológico de Cd. Victoria. Cd. Victoria, Tamaulipas, México, 304 pp.

Beier, M. (1954) Revision der Pseudophyllinen. Consejo Superior de Investigaciones Científicas-Trabajos del Instituto Español de Entomología. Madrid, España, 449 pp. Disponible en: https://www.worldcat.org/title/ revision-der-Pseudophyllinen/oclc/8569602 (última consulta julio 2019).

Beier, M. (1962) Orthoptera Tettigoniidae (Pseudophyllinae I). Das Tierreich, 73, 1-468.

Brunner von Wattenwyl, C. (1895) Monographie del Pseudophylliden. Herausgegeben Von der K. K. Zoologisch-Botanischen Gesellschaft in Wien, 45, 292 pp. Disponible en: https://www.zobodat.at/pdf/MON-E-ORTH_0007_0001-0282.pdf

Cigliano, M. M., Braun, H., Eades, D. C., Otte, D. (2019) Orthoptera Species File. Version 5.0/5.0. Disponible en: http://Orthoptera.SpeciesFile.org (última consulta en julio 2019).

Dutta, R., Tregenza, T., Balakrishnan, R. (2017). Reproductive isolation in the acoustically divergent groups of tettigoniid, Mecopoda elongata. PLoS ONE, 12 (11), e0188843. https://doi.org/10.1371/journal.pone.0188843

Heller, K. G., Orci, K. M., Grein, G., Ingrisch, S. (2004) The Isophya species of Central and Western Europe (Orthoptera: Tettigonioidea: Phaneropteridae). Tijdschrift Voor Entomologie, 147, 237258. https://doi.org/10.1163/22119434-900000153

Heller, K. G., Hemp, C., Ingrisch, S., Liu, C. (2015) Acoustic communication in Phaneropterinae (Tettigonioidea) - A global review with some new data. Journal of Orthoptera Research, 24 (1), 7 18. http://dx.doi.org/10.1665/034.024.0103

Heller, K. G., Ingrisch, S., Liu, C., Shi, F., Hemp, C., Warchałowska-Śliwa, E., Rentz, D. C. F. (2017) Complex songs and cryptic ethospecies: the case of the Ducetia japonica group (Orthoptera: Tettigonioidea: Phaneropteridae: Phaneropterinae). Zoological Journal of the Linnean Society, 181 (2), 286-307. https://doi.org/10.1093/zoolinnean/zlw019

Hollier, J., Maehr, M. D. (2012) An annotated catalogue of the type material of Orthoptera (Insecta) described by Carl Brunner von Wattenwyl deposited in the Muséum d'histoire naturelle in Geneva. Revue suisse de Zoologie, 119 (1), 27-75.

Kirby, W. F. (1906) A Synonymic Catalogue of Orthoptera (Orthoptera Saltatoria, Locustidae vel Acridiidae), Vol. II. Part I. Taylor and Francis, British Museum Natural History, London, 562 pp.

Montealegre-Zapata, F., Guerra, P. A., Morris, G. K. (2003) Panoploscelis specularis (Orthoptera: Tettigoniidae: Pseudophyllinae): extraordinary female sound generator, male description, male protest and calling signals. Journal of Orthoptera Research, 12 (2), 173-181. https://doi.org/10.1665/1082-6467(2003)012[0173:PSOTPE]2.0.CO;2

Piza, S. T. Jr (1980) Espécies novas de Pseudophyllinae especialmente do Brasil (Orthoptera, Tettigoniidae). Anais da Escola Superior de Agricultura "Luiz de Queiroz”, Universidade de São Paulo, 37 (1), 209-222. 
Fernández-Azuara et al.: Gongrocnemis (G.) munda señal acústica y redescripción

Ragge, D. R., Reynolds, W. J. (1998) The Songs of the Grasshoppers and Crickets of Western Europe. Colchester, Harley Books, 591 pp.

Robinson, D. J., Hall, M. J. (2002) Sound signalling in Orthoptera. Advances in Insect Physiology, 29, 151-278. https://doi.org/10.1016/S0065-2806(02)29003-7

Saussure de, H. (1859) Orthoptera Nova Americana. Diagnoses praeliminaris. Fam. Locustidae. Revue et Magasin de Zoologie, Paris, 2 (11),201-212.

Saussure de, H., Zehntner, L., Pictet, A. (1893-1899) Biología Centrali-Americana. Insecta, Orthoptera. Volume I. R.H. Porter, London, 463 pp.

Siegert, M. E., Römer, H., Hartbauer, M. (2013) Maintaining acoustic communication at a cocktail party: heterospecific masking noise improves signal detection through frequency separation. The Journal of Experimental Biology, 21, 4655-4665. https://doi.org/10.1242/jeb.089888

Stumpner, A., Angela, A., Schink, M., Gubert, S., Hugel, S. (2013) True katydids (Pseudophyllinae) from Guadeloupe: Acoustic signals and functional considerations of song production. Journal of Insect Science, 13, 157. Disponible en: http://www.insectscience.org/13.157 